\title{
Intraoperative Early Venous Filling Phenomenon as an Intrinsic Sign of the Local Hemodynamic Change after Revascularization Surgery in a Patient with Adult Moyamoya Disease: Implications of a Potential Arteriovenous Shunt
}

\author{
Kikutaro TOKAIRIN, ${ }^{1}$ Taku SugrYAmA, ${ }^{1}$ Masaki ITO,${ }^{1}$ and Miki FujIMURA ${ }^{1}$ \\ ${ }^{1}$ Department of Neurosurgery, Hokkaido University Graduate School of Medicine, Sapporo, \\ Hokkaido, Japan
}

\begin{abstract}
After revascularization surgery for patients with moyamoya disease (MMD), local and global hemodynamic changes occur intraoperatively and in the early postoperative period. Local cerebral hyperperfusion and watershed shift ischemia are well-known perioperative pathologies after revascularization for MMD, but early venous filling phenomenon is markedly rare. We report the case of a 19-year-old woman with hemorrhagic-onset MMD who presented with grand mal seizure and subarachnoid hemorrhage. She underwent superficial temporal artery (STA)middle cerebral artery (MCA) anastomosis combined with indirect pial synangiosis on the affected hemisphere. Intraoperatively, notable early arterial blood filling in the fine cortical vein was observed around the site of anastomosis right after the STA-MCA anastomosis under the surgical microscope and fluorescence indocyanine green video angiography. Recovery of consciousness after general anesthesia was normal, although she exhibited a focal seizure 1 hour later. Postoperative magnetic resonance imaging was not remarkable, and cerebral hemodynamics significantly improved in the acute stage after surgical revascularization. Considering the intrinsic vulnerability of the microvascular anatomy of MMD, the present case is notable because early venous filling was observed intraoperatively. This phenomenon suggests the existence of a potential arteriovenous shunt as an underlying pathology of MMD, but its implications in the early postoperative course should be further verified in a larger number of MMD patients undergoing surgical revascularization.
\end{abstract}

Keywords: moyamoya disease, direct revascularization surgery, arteriovenous shunt, reperfusion, cerebral hemodynamics

\section{Introduction}

Moyamoya disease (MMD) causes progressive steno-occlusive changes around the terminal portion of the internal carotid artery, resulting in the subsequent development of abnormal collateral vessels at the base of the brain. ${ }^{1,2)}$ The microvascular anatomy of the affected intracranial arteries in MMD patients

Received June 2, 2021; Accepted August 23, 2021

Copyright $@ 2021$ The Japan Neurosurgical Society This work is licensed under a Creative Commons AttributionNonCommercial-NoDerivatives International License. is characteristic due to its vulnerability from medial layer thinning and waving of internal elastic lamina. ${ }^{3}$ Blood-brain barrier (BBB) disruption around the affected vessels is also reported in MMD patients. ${ }^{4}$ The most effective treatment for MMD is revascularization surgery, but recent reports described potential complications after revascularization for MMD, such as postoperative ischemic or hemorrhagic stroke, or cerebral hyperperfusion (CHP) syndrome, during the postsurgical acute stage. ${ }^{5-7)}$ However, there are limited reports focusing on intraoperative findings regarding hemodynamic changes produced by direct anastomosis or superficial temporal artery (STA)-middle cerebral artery (MCA) anastomosis. 

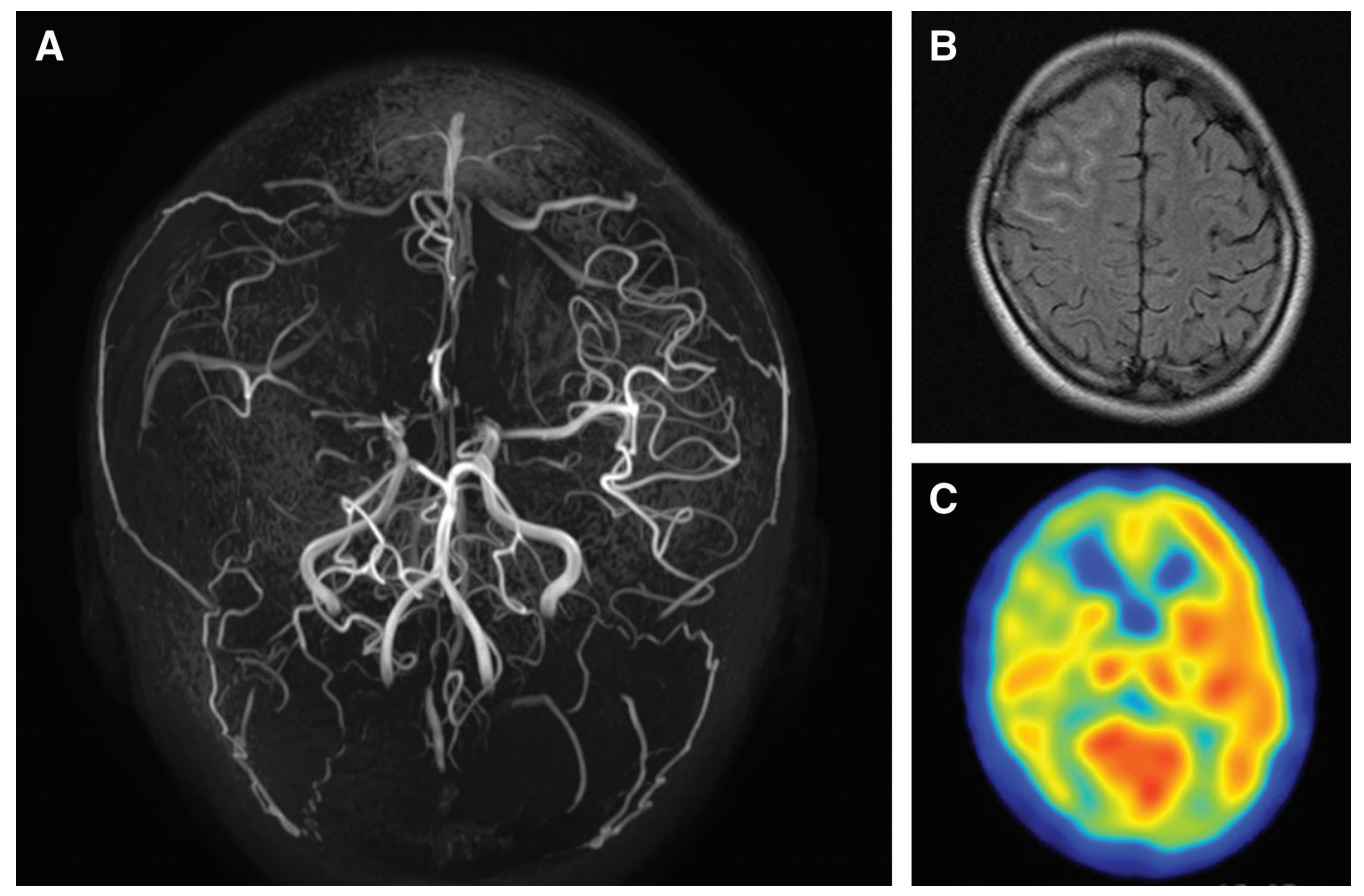

Figure 1 Preoperative brain MR imaging, MRA, and CBF findings. (A) Time-of-flight MR angiography showed bilateral stenosis in the arteries centered on the terminal portion of the intracranial internal carotid arteries, including anterior cerebral arteries (A1 segment) and right MCA, which indicated typical MMD. The signal of peripheral MCA was markedly decreased, and only a faint vascular shadow of Sylvian vein and middle meningeal artery were evident. (B) Fluid-attenuated inversion-recovery imaging demonstrated cortical SAH in the right frontal cortical sulci when the patient developed severe headache and grand mal seizure. (C) $\mathrm{N}$-isopropyl-p-[123] iodoamphetamine-SPECT showed decreased cerebral blood flow in the right cerebral hemisphere, especially in the frontal lobe cortex. CBF: cerebral blood flow, MCA: middle cerebral artery, MMD: moyamoya disease, MR: magnetic resonance, MRA: magnetic resonance angiography, SAH: subarachnoid hemorrhage, SPECT: single photon emission computed tomography.

In this case report, we describe the case of an adult MMD patient whose operative findings suggested an arteriovenous shunt immediate after anastomosis.

\section{Case Report}

A 19-year-old female presented with severe headache and grand mal seizure. She was brought to a local hospital, diagnosed with subarachnoid hemorrhage (SAH) by magnetic resonance imaging, and suspected of having MMD by magnetic resonance angiography (MRA) (Fig. 1A and 1B). The subarachnoid hematoma was localized in sulci of the right frontal lobe. She was admitted to our hospital for detailed examination 2 months after onset. She had a medical history of adjustment disorder. Digital subtraction angiography revealed stenosis of cerebral arteries around the terminal portion of internal carotid artery and moyamoya vessels at the base of the brain. These findings were observed bilaterally and were notable in the right hemisphere (Fig. 2A and 2B). She was diagnosed with MMD, and Suzuki's grade was judged as III and I in the right and left hemispheres, respectively. There was no arteriovenous malformation (AVM) or fistula by catheter angiography before surgery (Fig. 2A-2D). N-isopropyl-p-[ $\left.{ }^{[23} \mathrm{I}\right]$ iodoamphetamine-single photon emission computed tomography ( ${ }^{123}$ I-IMP-SPECT) revealed cerebral blood flow (CBF) reduction in the right hemisphere (Fig. 1C). She was indicated for right cerebral revascularization surgery to reduce the future risk of ischemic or hemorrhagic stroke.

She underwent combined revascularization surgery 3 months after onset. Under general anesthesia, we incised the skin just above STA parietal branch and performed frontotemporal craniotomy. The brain surface, arteries, and veins were typical of $\mathrm{MMD}$, as shown in Fig. 3A. There was no arteriovenous shunt microscopically. A cortical branch of the right MCA on frontal lobe was thought to be ideal as the anastomosis recipient based on its diameter and color tone (Fig. 3A). The harvested STA frontal branch was cut into a "fish mouth" shape and anastomosed to the cortical artery using 10-0 nylon 

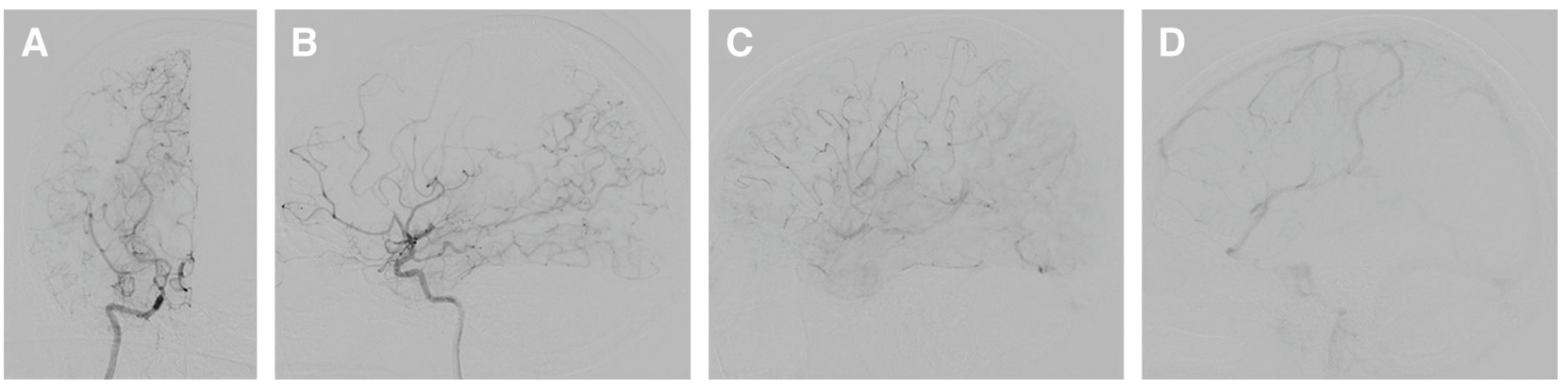

Figure 2 Catheter angiography before the revascularization surgery. (A) Anterior-posterior and (B) lateral views on right ICA angiography demonstrated arterial narrowing in the arteries centered on the terminal portion of the intracranial ICA with intensification of the basal moyamoya vessels. Sprouting angiogenesis was observed at the periphery of the middle and posterior cerebral artery boundary (B). Neither major nor peripheral arterial aneurysm was observed. Arterial (B), capillary (C), and venous phase (D) of angiography showed no arteriovenous shunt in the frontal lobe. ICA: internal carotid artery.

sutures. The clamp time of the MCA during anastomosis was 24 minutes. Anastomosis was confirmed patent without blood leakage from the seam, and there were no microscopic blood clots inside (dotted circle in Fig. 3B). However, some veins were red in color, reflecting inappropriate arterial blood inflow (black arrowheads in Fig. 3B), in contrast to the superficial Sylvian vein that was dark in color (white arrows in Fig. 3B). Indocyanine green (ICG) video angiography revealed the apparent early inflow of arterial blood from the anastomosis site (dotted circle in Fig. 3C) toward the adjacent cortical veins (black arrowheads in Fig. 3D) and its downstream superficial Sylvian vein (white arrows in Fig. 3E). The direct anastomosis was completed by a single bypass, and indirect bypass was performed in an encephalo-duro-myo-synangiosis manner.

The patient awoke from general anesthesia, and there were no abnormal findings on computed tomography just after the surgery (data not shown); however, she exhibited a partial seizure 1 hour after the surgery. Diazepam was injected and the seizure ceased. She was started on the anti-epileptic drugs levetiracetam and lacosamide. We initiated strict blood pressure (BP) control of a systolic BP of 100-130 $\mathrm{mmHg}$ and minocycline injection for 7 days to prevent CHP. ${ }^{8)}$ Postoperative ${ }^{123}$ I-IMPSPECT on the postoperative day (POD) 1 and 7 demonstrated significant improvement of $\mathrm{CBF}$ without evidence of local CHP (Fig. 4A). Postoperative MRA on POD 2 revealed patency of the bypass (Fig. 4B and 4C). The patient had no complications other than the partial seizure. She was discharged on POD 15 and had no stroke or transient ischemic attack 3 months after the surgery. The patient and her parent provided written informed consent to publish the case report.

\section{Discussion}

We described a case of MMD with a potential arteriovenous shunt adjacent to the anastomosis site, which became evident just after STA-MCA anastomosis intraoperatively. There are only limited reports on the de novo formation of an arteriovenous shunt after STA-MCA anastomosis in MMD patients. Peeters and colleagues reported an MMD patient who developed dural and pial arteriovenous fistula (AVF) in the operative field 8 months after STA-MCA anastomosis that spontaneously resolved within 2 years. ${ }^{9)}$ We also reported the de novo formation of occipital AVM at an area outside the operative field 4 years after bilateral revascularization surgeries for child-onset MMD. ${ }^{10)}$ This suggests that delayed formation of an arteriovenous shunt or AVM can occur after revascularization surgery for MMD patients. However, de novo formation of an arteriovenous shunt immediately after STA-MCA bypass, as in the present case, has not been reported previously. The intraoperative early venous filling phenomenon near the site of STA-MCA anastomosis in our case suggests arteriovenous shunts as an underlying pathology of MMD.

The exact mechanism underlying the de novo formation of the potential arteriovenous shunt immediately after STA-MCA bypass in our case is undetermined. Considering its development immediately after STA-MCA anastomosis near the site of the anastomosis, it is conceivable that the potential arteriovenous shunt, which was angiographically absent before surgery, became evident after local hemodynamic change by direct revascularization in our patient. Increased angiogenesis under the chronic ischemic condition in MMD patients may play a role, at least in part, in the association with 

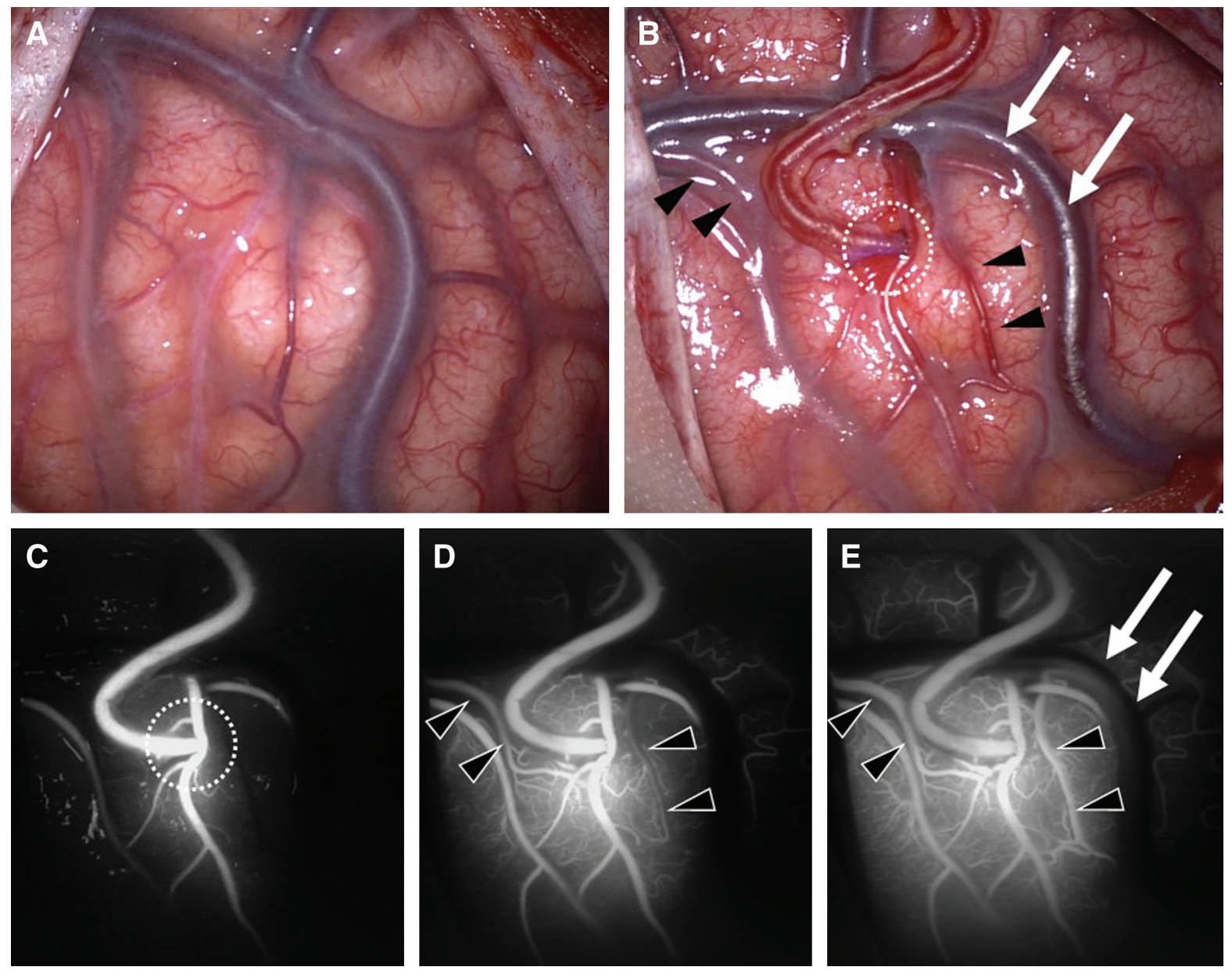

Figure 3 Intraoperative photographs during STA-MCA anastomosis. (A) The brain surface view of the right frontal lobe before the anastomosis showed dilated pial arteries, cloudiness of the arachnoid membrane, and xanthochromic spots in the cortical sulci, indicating hemosiderin deposits due to prior SAH. (B) Right after the STA-MCA anastomosis, fine cortical veins (black arrowheads) next to the site of anastomosis (dotted circle) turned arterial reddish in color. A part of the downstream superficial Sylvian vein (white arrows) also turned reddish, which indicated earlier arterial blood filling to the vein. (C) An earlier arterial phase of ICG video angiography demonstrated that arterial blood flow first came into the site of anastomosis (dotted circle) and toward the recipient MCA via the donor STA. (D) Subsequently, still in the early arterial phase, fluorescent ICG signal appeared in the fine cortical veins (black arrowheads), indicating earlier arterial blood filling to the vein. (E) In the latter arterial phase, early arterial blood filling near the site of anastomosis became more prominent (black arrowheads) and the laminar ICG signal flow was in part of the downstream superficial Sylvian vein (white arrows). ICG: indocyanine green, MCA: middle cerebral artery, SAH: subarachnoid hemorrhage, STA: superior temporal artery.

potential arteriovenous shunts. This hypothesis is based on the previous observation by Osada and colleagues about the relatively common association of pial AVF with dural AVF, in which the authors hypothesized that hemodynamic compromise by venous congestion and subsequent activation of angiogenesis ultimately lead to the appearance of potential pial AVF. ${ }^{11)}$ Indeed, angiogenic factors, such as vascular endothelial growth factors and matrix metalloproteinase-9, are involved in both MMD and cerebral vascular malformations. ${ }^{12,13)}$ Alternatively, recent intraoperative analysis by fluorescein video angiography strongly suggested that the BBB structure is significantly compromised in MMD patients ${ }^{4}$ and BBB dysfunction is also a characteristic pathology of cerebral vascular malformations. ${ }^{14,15)}$

The clinical implications of the intraoperative local formation of an arteriovenous shunt immediately after STA-MCA anastomosis should be further verified in future studies, although the postoperative course was generally favorable after the improvement of cerebral hemodynamics in our case. STA-MCA anastomosis is a standard surgical procedure for 

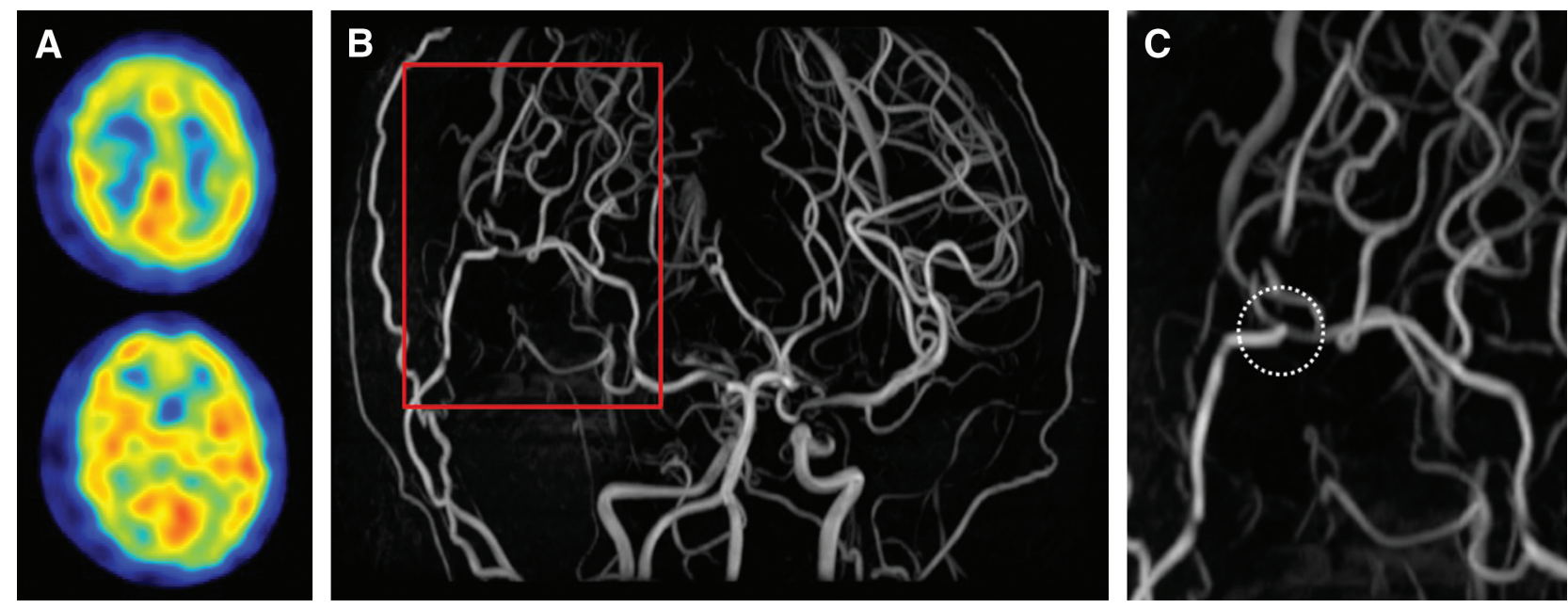

Figure 4 Postoperative brain MRA and CBF findings. (A) N-isopropyl-p-[123I] iodoamphetamine-SPECT on POD 1 showed increased CBF in the right hemisphere compared with the preoperative status. (B) MRA on POD 2 demonstrated apparently patent STA-MCA anastomosis (dotted circle). (C) Higher magnification at the site of anastomosis is shown in Panel B. CBF: cerebral blood flow, MCA: middle cerebral artery, MRA: magnetic resonance angiography, POD: postoperative day, SPECT: single photon emission computed tomography, STA: superior temporal artery.

MMD patients, but dynamic changes in local cerebral hemodynamics by surgical revascularization can cause intrinsic perioperative pathologies such as local $\mathrm{CHP}^{16)}$ and/or characteristic hemodynamic ischemia, the so-called watershed shift phenomenon. ${ }^{17,18)}$ Machida et al. reported that cortical venous redness after STA-MCA anastomosis is significantly correlated with the postoperative CBF increase in MMD patients. ${ }^{19)}$ Taken together with previous reports about the usefulness of intraoperative monitoring of the cortical hemodynamic changes by ICG video angiography or by thermography after STA-MCA anastomosis for predicting postoperative CHP syndrome in MMD patients,,$^{20,21)}$ the impact of the formation of a potential arteriovenous shunt immediately after STA-MCA anastomosis on the postoperative hemodynamics and clinical course in a larger number of MMD patients undergoing surgical revascularization is of interest.

Our study has several limitations. First, we did not perform intraoperative ICG video angiography before the bypass procedure, which could have convinced the intraoperative local formation of an arteriovenous shunt immediately after STA-MCA anastomosis. Second, long-term outcome of the newly formed arteriovenous shunt is undetermined because of the very short follow-up period of this patient. Follow-up catheter angiography in this patient would be very useful to answer this important question. Finally, we do not completely rule out the possibility that STA-MCA anastomosis normalized the arterial transit time only in the local cortical area adjacent to the bypass when the surrounding cortex still manifested as the delayed arterial transit time. In such condition, rapidly normalized arterial transit time could lead to the decrease in local oxygen extraction fraction and consequently cause venous redness at the site of the anastomosis.

\section{Conclusions}

We reported an adult MMD patient who intraoperatively presented with the early formation of a potential arteriovenous shunt near the site of revascularization just after STA-MCA anastomosis. This phenomenon suggests that an arteriovenous shunt is an underlying pathology of MMD, but its implications in the early postoperative course should be further verified in a larger number of MMD patients undergoing surgical revascularization.

\section{Funding}

This study was supported by JSPS KAKENHI Grant Number 20K09362 (M.F.). The authors have no conflicting remarks.

\section{Conflicts of Interest Disclosure}

The authors have nothing to declare. The authors registered online Self-Reported COI Disclosure Statement Forms through the website for Japan Neurosurgical Society members. 


\section{References}

1) Suzuki J, Kodama N: Moyamoya disease-a review. Stroke 14: 104-109, 1983

2) Kuroda S, Houkin K: Moyamoya disease: current concepts and future perspectives. Lancet Neurol 7: 1056-1066, 2008

3) Takagi Y, Kikuta K, Nozaki K, Hashimoto N: Histological features of middle cerebral arteries from patients treated for moyamoya disease. Neurol Med Chir (Tokyo) 47: 1-4, 2007

4) Narducci A, Yasuyuki K, Onken J, Blecharz K, Vajkoczy P: In vivo demonstration of blood-brain barrier impairment in moyamoya disease. Acta Neurochir (Wien) 161: 371-378, 2019

5) Tokairin K, Kazumata K, Uchino H, et al.: Postoperative intracerebral hemorrhage after combined revascularization surgery in moyamoya disease: profiles and clinical associations. World Neurosurg 120: e593-e600, 2018

6) Fujimura M, Mugikura S, Kaneta T, Shimizu H, Tominaga $\mathrm{T}$ : Incidence and risk factors for symptomatic cerebral hyperperfusion after superficial temporal artery-middle cerebral artery anastomosis in patients with moyamoya disease. Surg Neurol 71: 442-447, 2009

7) Kazumata K, Ito M, Tokairin K, Ito Y, Houkin K, Nakayama N, Kuroda S, Ishikawa T, Kamiyama H: The frequency of postoperative stroke in moyamoya disease following combined revascularization: a single-university series and systematic review. $J$ Neurosurg 121: 432-440, 2014

8) Fujimura M, Inoue T, Shimizu H, Saito A, Mugikura S, Tominaga T: Efficacy of prophylactic blood pressure lowering according to a standardized postoperative management protocol to prevent symptomatic cerebral hyperperfusion after direct revascularization surgery for moyamoya disease. Cerebrovasc Dis 33: 436-445, 2012

9) Peeters SM, Colby GP, Guivatchian E, Sun MZ, Tateshima S, Wang AC: Spontaneous resolution of dural and pial arteriovenous fistulae arising after superficial temporal artery to middle cerebral artery bypass for moyamoya disease. World Neurosurg 142: 404-407, 2020

10) Fujimura M, Kimura N, Ezura M, Niizuma $K$, Uenohara H, Tominaga T: Development of a de novo arteriovenous malformation after bilateral revascularization surgery in a child with moyamoya disease. $J$ Neurosurg Pediatr 13: 647-649, 2014

11) Osada T, Krings T: Intracranial dural arteriovenous fistulas with pial arterial supply. Neurosurgery 84: 104-115, 2019

12) Fujimura $M$, Watanabe $M$, Narisawa A, Shimizu H, Tominaga T: Increased expression of serum matrix metalloproteinase-9 in patients with moyamoya disease. Surg Neurol 72: 476-480; discussion 480, 2009

13) Kang HS, Kim JH, Phi JH, et al.: Plasma matrix metalloproteinases, cytokines and angiogenic factors in moyamoya disease. J Neurol Neurosurg Psychiatry 81: 673-678, 2010

14) Clatterbuck RE, Eberhart CG, Crain BJ, Rigamonti D: Ultrastructural and immunocytochemical evidence that an incompetent blood-brain barrier is related to the pathophysiology of cavernous malformations. $J$ Neurol Neurosurg Psychiatry 71: 188-192, 2001

15) Tu J, Stoodley MA, Morgan MK, Storer KP: Ultrastructural characteristics of hemorrhagic, nonhemorrhagic, and recurrent cavernous malformations. $J$ Neurosurg 103: 903-909, 2005

16) Fujimura $M$, Shimizu $H$, Inoue $T$, Mugikura $S$, Saito A, Tominaga T: Significance of focal cerebral hyperperfusion as a cause of transient neurologic deterioration after extracranial-intracranial bypass for moyamoya disease: comparative study with non-moyamoya patients using N-isopropyl-p-[(123)I]iodoamphetamine single-photon emission computed tomography. Neurosurgery 68: 957-964; discussion 964-965, 2011

17) Hayashi T, Shirane R, Fujimura M, Tominaga T: Postoperative neurological deterioration in pediatric moyamoya disease: watershed shift and hyperperfusion. J Neurosurg Pediatr 6: 73-81, 2010

18) Tashiro R, Fujimura $M$, Kameyama $M$, et al.: Incidence and risk factors of the watershed shift phenomenon after superficial temporal artery-middle cerebral artery anastomosis for adult moyamoya disease. Cerebrovasc Dis 47: 178-187, 2019

19) Machida T, Higuchi $Y$, Nakano S, et al.: Cortical venous redness represents tissue circulation status in patients with moyamoya disease. Stroke 48: 16651667, 2017

20) Nakagawa A, Fujimura M, Arafune T, Sakuma I, Tominaga T: Clinical implications of intraoperative infrared brain surface monitoring during superficial temporal artery-middle cerebral artery anastomosis in patients with moyamoya disease. J Neurosurg 111: 1158-1164, 2009

21) Uchino H, Kazumata K, Ito M, Nakayama N, Kuroda S, Houkin K: Intraoperative assessment of cortical perfusion by indocyanine green videoangiography in surgical revascularization for moyamoya disease. Acta Neurochir (Wien) 156: 1753-1760, 2014

Corresponding author: Miki Fujimura, MD, PhD Department of Neurosurgery, Hokkaido University Graduate School of Medicine, Kita15 Nishi7, Kita-ku, Sapporo, Hokkaido 060-8638, Japan. e-mail: fujimur@med.hokudai.ac.jp 